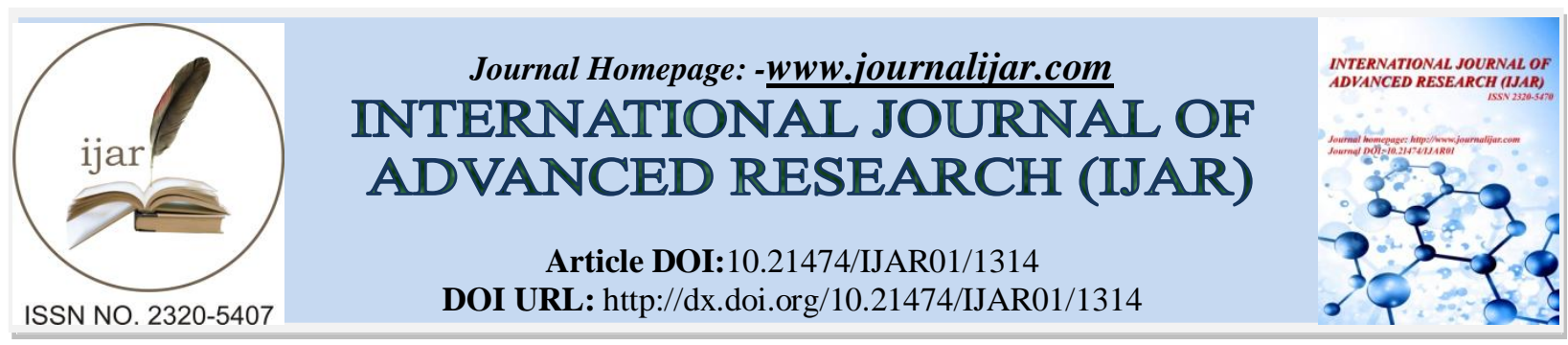

RESEARCH ARTICLE

\title{
IDENTIFICATION AND CLASSIFICATION OF FABRIC DEFECTS.
}

\author{
Karanveer Singh $^{1}$ and Jaspreet Kaleka ${ }^{2}$. \\ 1. M.tech Student, UCoE, Punjabi University, Patiala, India. \\ 2. Assistant Professor, UCoE, Punjabi University, Patiala, India.
}

\section{Manuscript Info}

Manuscript History

Received: 18 June 2016

Final Accepted: 15 July 2016

Published: August 2016

Key words:-

Fabric defects, Fourier analysis, Gabor filters, Textured Material.

\begin{abstract}
Fabric is widely used nowadays in every aspect of our life but many a times we encounter different defects in fabrics. So, to detect these defects in the fabric different types of techniques are used at the industrial level to deliver a refined product of the flawless fabric. Automated Inspection is preferred as many defects may get ignored during the human analysis. In this review paper, I will be concentrating on the different techniques used to segment the defected part of an image from the rest of the image.
\end{abstract}

Copy Right, IJAR, 2016,. All rights reserved.

\section{Introduction:-}

Fabric, being a widely used material in daily life, is manufactured with textile fibers. Textile fibers are made up of natural elements such as cotton or wool; or a composite of different elements such as wool and nylon or polyester. Fabric defect detection is a quality control process that aims at identifying and locating defect of fabric. Human inspection is the traditional means to assure the quality of fabric but fine defects are often ignored. Therefore, inspection done automatically of fabric defects becomes a natural way to improve fabric quality and reduce labor costs. Defect detection in web materials normally depends upon identification of regions that differ from a uniform background. The textured materials can be further divided into uniform, random, or patterned textures.

\section{Fabricdefects:-}

It has been estimated [1] that the price of fabrics is reduced by $45 \%-65 \%$ due to the presence of defects. The quality of the fabric gets affected by quality of yarn and/or loom defects. The poor quality of raw materials and poor condition of yarn leads to yarn quality defects and effects such as color or width inconsistencies, hairiness, slubs, broken ends, etc. The tests done on the quality of yarns are the output obtained at spinning-mills. Quality test performed on looms and knitting machines needs interruption of the weaving process. These test runs tend to be smaller and may not identify recurring fabric defects that are generated due to sinusoidal occurring inconsistencies in the weaving machines. The fabric defects resulting from variations in the tension of one or more yarn strands are generally misread as the defects resulting from poor yarn quality. The population of fabric defects may vary dynamically as small changes in the weaving process can result in an entirely new class of fabric defects. Below is a brief of some most identified defects along with their reasons.

Corresponding Author:-Karanveer Singh

Address:-M.tech Student, UCoE, Punjabi University, Patiala, India. 


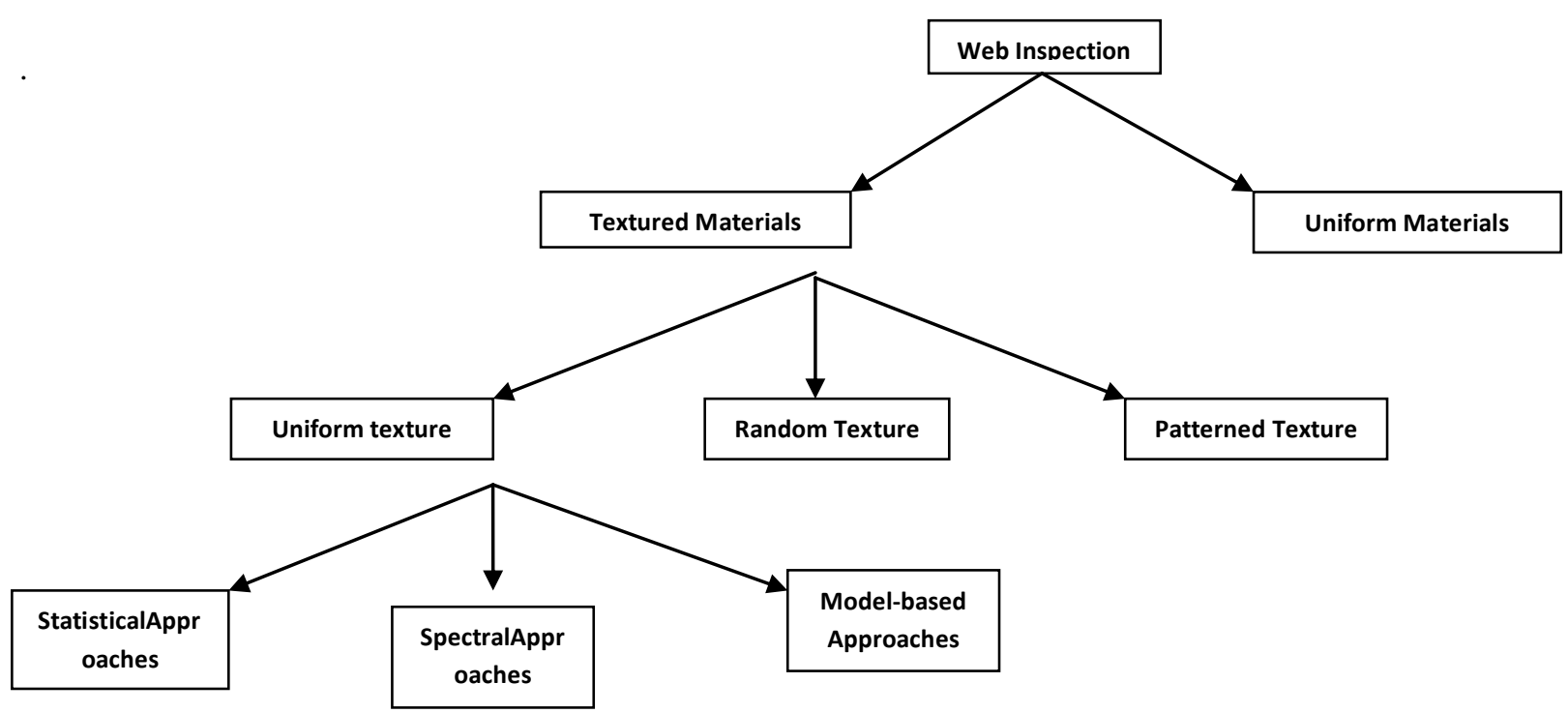

Figure 1:- Classification of a web inspection problem based on the nature of the surface. [4].

Table 1:-Types of major fabric defects.

\begin{tabular}{|c|c|c|}
\hline Defect type & Definition & Reasons \\
\hline Floats & $\begin{array}{l}\text { A portion of a yarn in a fabric that extends } \\
\text { or floats, unbound, over two or more } \\
\text { adjacent ends or pith }\end{array}$ & $\begin{array}{l}\text { It is caused by missing of interlacement of } \\
\text { two series of threads }\end{array}$ \\
\hline Holes & $\begin{array}{l}\text { A fabric area free of both warp and weft } \\
\text { threads }\end{array}$ & $\begin{array}{l}\text { It is a mechanical fault caused by a } \\
\text { broken machine part }\end{array}$ \\
\hline Oil stains & Fabric area contains oil spots & $\begin{array}{l}\text { It is caused by too much oiling on loom } \\
\text { parts or from other external sources }\end{array}$ \\
\hline Slubs & A local uneven fabric thickness & $\begin{array}{l}\text { It is caused by an extra piece of yarn that } \\
\text { is woven into fabric. It can also be caused } \\
\text { by thick places in the yarn or by fly waste } \\
\text { being spun in yarn during the spinning } \\
\text { process }\end{array}$ \\
\hline Knots & $\begin{array}{l}\text { A fabric place where two ends of yarn } \\
\text { have been tied together and the tails of the } \\
\text { knot are protruding from the surface }\end{array}$ & $\begin{array}{l}\text { It is caused by tying spools of yarn ends } \\
\text { together }\end{array}$ \\
\hline Mis-end & $\begin{array}{l}\text { A warp thread is absent in the fabric for a } \\
\text { short or long distance }\end{array}$ & $\begin{array}{l}\text { It is due to incorrect warping or by a } \\
\text { broken warp thread that never replaced by } \\
\text { another one }\end{array}$ \\
\hline Mis-pick & $\begin{array}{l}\text { A weft thread is absent in the fabric for a } \\
\text { short or long distance }\end{array}$ & $\begin{array}{l}\text { It is caused by incorrect picking or if the } \\
\text { weaver restarted the loom after any } \\
\text { stoppage without adapting the position for } \\
\text { the new insertion }\end{array}$ \\
\hline
\end{tabular}


Defects can be classified into any number of terms. Below is visual view of some defects according to Shanbhag et al. [3]

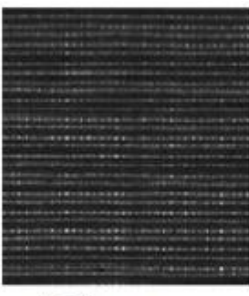

a) Tripe- warp

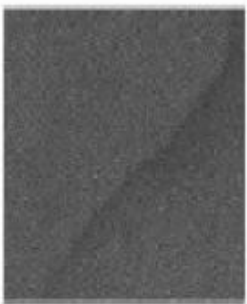

d) Water damage

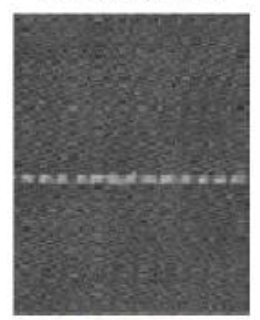

g) Foriegn Fiber

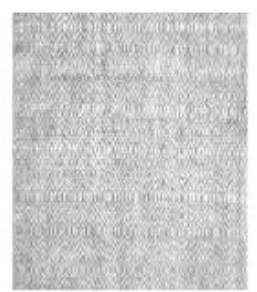

b) Misspick

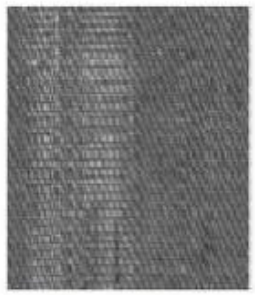

e) Netting Multipliers

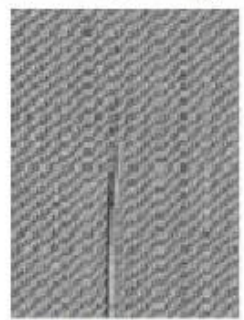

h) Overshoot

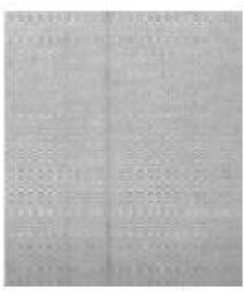

c) Slackend

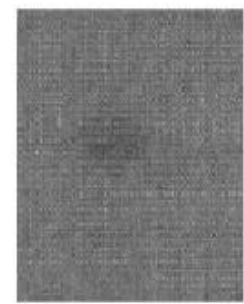

f) Oil spot

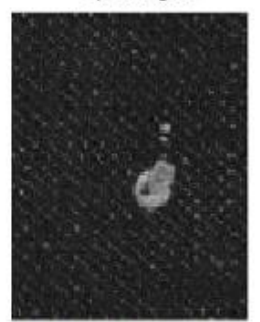

i) Kinky Filling

Figure 2:- Images of fabrics with different types of defects [3]

\section{Approaches used in texture based defect identification system:-}

\section{Statistical Approaches:-}

Statistical methods indirectly represent the texture in according to the non-deterministic properties of the distributions and relation between the gray levels of an image. The objective of defect detection is to differentiate the inspected image into well-defined regions of statistical behavior. The analysis of defect-free regions is stationary, and these obtained regions extend over a notable portion of the inspection images [4].

1. Defect Detection Using Fractal Dimension (FD): Conci and Proenca [5] have implemented the differential box counting method with few modifications to minimize computational complexity and to enhance efficiency. They used the estimate of FD on inspection images to detect fabric defects.

2. Defect detection using Cross-Correlation: For detecting features in one image that appear in another providing a direct and correct measure of similitude between two images, is called Correlation. Any noticeable variation in the values of obtained measurements indicates the presence of a defect.

3. Multilevel Thresholding Approach: This approach is used to inspect uni-coloured fabrics without consideration of texture. The defective regions are segmented by using two thresholding methods.

4. Optimal filter design Approach: This approach concerns the determination of a filter that provides the largest discrimination between two textures. The classifications were made on the basis of either gray level statistics or morphological operations. The autocorrelation function was used to identify fabric structural-repeat units to carry out either statistical or morphological computations.

5. Independent Component Analysis: ICA wishes to find a linear transformation of the original data such that the new depiction is one that reduces the reliance of the components present in the representation on the statistical basis. The representation achieved by ICA ease the analysis of the data experienced in such fields like, data compression, pattern recognition, de-noising [6].

6. Statistical Moments Approach: In these techniques a statistical sample is gathered by preferring large windows. The algorithm of obtaining texture has characteristics directly from the gray-level image by evaluating the 
moments in local regions has successfully divided the binary images having textures with iso-second order statistics as well as a number of gray level texture images.

7. Rank order function Approach: This approach is based on histogram analysis which is a simple statistical approach. It is given by the sequence of gray levels in the histogram when this sequence is sorted in the ascending order. There exists 1:1 correspondence between the rank function and the related histogram, which does not exist between histogram and the image. Harwood et al. [7] found that, local rank-order correlations of images with Laws' masks could perform better than the basic convolutions, for suitable image and mask sizes.

\section{Spectral Approaches:-}

Spectral approaches hold a big portion of the latest computer vision research work. It analyzes the textured images in the spatial frequency domain. The primary objectives of spectral approaches are to extract texture primitives or generalize the spatial placement rules. The spectral approaches are summarized in the following sections.

1. Fourier analysis Approach: Faultless fabric is a regular global texture and Fourier transform can be applied to check the fabric having spatial frequency spectrum. When a fabric is defected, its regular form is changed so that the corresponding intensity at some particular positions of the frequency spectrum would differ. This indicates the textured image in terms of frequency components. Fourier techniques have advantageous properties of noise immunity, translation invariance and the optimal characterization of the periodic features. An approach based on Fourier transform has been described to detect the structural defect in fabric. The simulated models are used to understand the behavior of frequency spectrum. The Fourier transform of textile fabric can be obtained in optical domain by using lenses and spatial filters.

2. Defect detection using Wigner distributions: The Wigner distribution function is Fourier-like but offers better co-joint resolution than Gabor or difference of Gaussians for co-joint spatial and spatial-frequency image representation. Song et al. [8] have used a computational approximation to the Wigner distribution, i.e., pseudoWigner distribution, to demonstrate the detection of cracks in complex background textured materials.

3. Defect Detection Using Optimized FIR Filters: A potential solution to detect fabric defect that produce very subtle intensity transitions is to employ optimal finite impulse response (FIR) filters. The biggest advantage of FIR filters is that they can implement any impulse response, of finite length. The optimization offers the potential of large feature separation between the defect-free and the defective regions of the filtered image.

\section{Model Based Approaches:-}

This approach tries to capture the process that generates the texture. This is done by determining the parameters of a pre-defined model. Texture is a complex pictorial pattern and defined by a stochastic or a deterministic model. The real textures can be represented as stochastic processes, and textured images can be noticed as the realizations or the obtained samples on the image space are from parametric probability distributions. These approaches often require that the image features at different levels of specificity or detail match one of possible models of different image classes.

Model based texture analysis such as Fractal model and Markov are based on the construction of an image that can be used for describing texture and synthesizing it [9]. These methods describe an image as a probability model or as a linear combination of a set of basic functions. The Fractal model is useful for representing certain natural textures that statistically have quality of irregularity at different scales and also for texture analysis and discrimination. Pixelbased models identify an image as a collection of pixels, whereas Region-based models as a set of sub patterns. There are different types of models based on the different neighborhood systems and noise sources, are onedimensional time-series models, Auto Regressive (AR), Moving Average (MA) and Auto Regressive Moving Average (ARMA). Random field models examine spatial variations in two dimensions, global random and local random. Global random field models consider the whole image as a realization of a random field, and local random field models infer relationships of intensities in small neighborhoods. The commonly used class of local random field models is Markov models, where the conditional probability of the intensity of a given pixel relies only on the intensities of the pixels in its neighborhood (the so-called Markov neighbors).

\section{Conclusion:-}

In this paper, the different approaches explained such as Statistical Approaches, Spectral Approaches, Model based Approaches. The main aim of this paper is to distinguish between the defected part and the rest the part of an image, of fabric from which the defect has to be defected. The Statistical Approach is used to differentiate the inspected image into well-defined regions of statistical behavior whereas the Spectral Approaches works on the spatial frequency domain of the textured image. Model based Approach is done by determining the parameters of a pre- 
defined model. But these techniques can be complex and need the manual differentiation of the textured image so as to classify it into defected part and the rest of the part of the image considered.

\section{References:-}

1. K. Srinivasan, et al. (1992), "FDAS: A knowledge-based frame detection work for analysis of defects in woven textile structures," J. Text. Inst., vol. 83, no. 3, pp. 431-447.

2. Priyanka M. Shanbhag, et al. (2012), "Overview: Methods of Automatic Fabric Defect Detection" Published in Global Journal of Engineering, design and technology vol. 1(2) Page no. 42-46.

3. Kumar (2008), "Computer-Vision-Based Fabric Defect Detection: A Survey," IEEE Trans. Ind. Electron., vol. 55, no. I, pp. 348-363.

4. Gedziorowski M., et al. (1995), "Programmable optical digital processor for rank order and morphological filtering", Optics Communications, Vol. 119, Issues 1-2, pp. 207-217.

5. A. Serdaroglu, A. Ertuzun, et al. (2006), "Defect detection in textile fabric images using wavelet transforms and independent component analysis", Pattern recognition \& image analysis, vol.16, no.1, pp.61-64.

6. Thilepa R., et al. (2010) "A Paper on Automatic Fabrics Fault Processing Using Image Processing Technique in MATLAB”, Signal \& Image Processing: An International Journal (SIPIJ), Vol. 1, No. 2, pp. 88 - 99.

7. K. Y. Song, e al. (1995), "Texture crack detection,” Mach. Vis. Appl., vol. 8, no. 1, pp. 63-76.

8. Taun Anh Pham (2010), "Optimization of texture feature extraction algorithm" master thesis, Delft University of Technology. 